\title{
AVALIAÇÃO DA QUALIDADE DE VIDA DE PORTADORES DE HIV/AIDS: UMA VISÃO GERAL DOS INSTRUMENTOS WHOQOL- HIV E WHOQOL-HIV-BREF
}

\author{
Bruno Pedroso, Universidade Estadual de Campinas - UNICAMP, Campinas, São Paulo - \\ Brasil \\ Gustavo Luis Gutierrez, Universidade Estadual de Campinas - UNICAMP, Campinas, São \\ Paulo - Brasil \\ Edison Duarte, Universidade Estadual de Campinas - UNICAMP, Campinas, São Paulo - \\ Brasil \\ Luiz Alberto Pilatti, Universidade Tecnológica Federal do Paraná - UTFPR, Ponta \\ Grossa, Paraná - Brasil \\ Claudia Tania Picinin, Universidade Positivo - UP, Curitiba, Paraná - Brasil
}

\section{RESUMO}

O objetivo do presente trabalho é clarificar o mecanismo pré-estabelecido pelo Grupo WHOQOL-HIV para o cálculo dos escores dos instrumentos WHOQOL-HIV e WHOQOL-HIV-bref. Para tanto foi realizada a transcrição textual das sintaxes dos referidos instrumentos, descrevendo em minúcias a depuração de tais sintaxes. De forma adicional, foram propostas ferramentas alternativas para o cálculo automatizado dos escores e estatística descritiva de tais instrumentos. Conclui-se que a dificuldade de interpretação das sintaxes e a utilização do SPSS para calcular os resultados dos instrumentos WHOQOL constituem fatores limitantes na sua utilização e que estes podem ser suprimidos por meio do entendimento das sintaxes e utilização de formas alternativas para o cálculo dos resultados destes instrumentos.

Palavras-Chave: Qualidade de vida; Instrumentos de avaliação; HIV/AIDS; WHOQOLHIV; WHOQOL-HIV-bref.

\section{AVALUACIÓN DE LA CALIDAD DE VIDA DE POORTADORES DE HIV/AIDS: UNA VISIÓN GENERAL DE LOS INSTRUMENTOS WHOQOL-HIV Y WHOQOL HIV BREF}

\section{RESUMEN}

El objetivo del presente trabajo es aclarar el mecanismo pre-establecido por el grupo WHOQOL-HIV y WHOQOL-HIV BREF. Para tanto fue realizada la transcripción textual de las sintaxis de los referidos instrumentos, describiendo em detalles la depuración de tales sintaxis. De forma adicional, fueron propuestas herramientas alternativas para el cálculo automático de los escores e estadistícas de tales instrumentos. Concluye que la dificultad para interpretar las sintaxis y la utilización del SPSS para calcular los resultados de los instrumentos WHOQOL constituyen factores limitadores para su uso y que estos 
pueden ser suprimidos por la comprensión de las sintaxis y utilización de formas alternativas para el cálculo de los resultados de estos instrumentos.

Palabras-Claves: Calidad de vida, Instrumentos de avaluación, HIV/AIDS, WHOQOLHIV, WHOQOL-HIV-bref.

\title{
EVALUATION OF THE QUALITY OF LIFE OF PEOPLE LIVING WITH HIV/AIDS: A GLOBAL VIEW OF WHOQOL-HIV AND WHOQOL-HIV-BREF INSTRUMENTS
}

\begin{abstract}
The objective of this paper is to clarify the mechanism pre-established by the WHOQOLHIV Group for the score calculation of WHOQOL-HIV and WHOQOL-HIV-bref instruments. For that, it was performed the textual transcription of the instruments syntaxes, describing in details the depuration of such syntaxes. It was proposed alternatives tools for the calculation of scores and descriptive statistics of such instruments. It concludes that the syntaxes interpretation difficulty and SPSS's utilization to calculate the WHOQOL's instruments results constitute limiting factors in its utilization and these can be suppressed by understanding of the syntaxes and utilization of alternative forms for the results calculation of such instruments.
\end{abstract}

Key-Words: Quality of life; Measurement instruments; HIV/AIDS, WHOQOL-HIV, WHOQOL-HIV-bref. 


\section{INTRODUÇÃO}

A avaliação da qualidade de vida de portadores de HIV e AIDS tem se tornado crescente. Entre 1995 e 2003, mais de 300 artigos sobre o tema foram publicados. Esse fato instiga os pesquisadores a questionarem a existência de instrumentos de avaliação apropriados. Praticamente todos os instrumentos existentes até 2003 haviam sido desenvolvidos nos EUA. $^{1}$

Para a aplicação desses instrumentos em países onde o inglês não é o idioma vernáculo, os instrumentos eram submetidos a traduções literais, sem a preocupação de uma adaptação cultural. Frente a essa perspectiva, surgiu a proposta de elaboração de um instrumento desenvolvido a partir de vários centros, situados em diferentes países. ${ }^{1}$

O fato de não haver um consenso sobre o conceito de qualidade de vida constitui um grande problema no desenvolvimento de instrumentos de avaliação da qualidade de vida, ao passo que não é possível afirmar, com clareza, quais elementos estes instrumentos estão mensurando. ${ }^{2}$

Partindo dessa premissa, o ponto inicial para a construção do instrumento de avaliação da qualidade de vida da Organização Mundial da Saúde (OMS), foi conceituar qualidade de vida. No conceito adotado, qualidade de vida é entendida como a "percepção do indivíduo de sua posição na vida no contexto da cultura e sistema de valores nos quais ele vive e em relação aos seus objetivos, expectativas, padrões e preocupações". ${ }^{2}$ (p. 25).

Frente a esse conceito, a OMS empenhou-se na construção dos instrumentos WHOQOL, que avaliam a qualidade de vida, tanto de forma global, como o WHOQOL-100 e o WHOQOL-bref, como também em função de aspectos específicos, como o WHOQOLHIV, WHOQOL-HIV-bref, WHOQOL-OLD e WHOQOL-SRPB. Os instrumentos WHOQOL-HIV e WHOQOL-HIV-bref, direcionados para a avaliação da qualidade de vida dos portadores do HIV, são objeto de estudo da presente investigação. 
Partindo da constatação de que $95 \%$ das pessoas infectadas com o HIV não residiam nos EUA, mas nos países em desenvolvimento da Ásia, America Latina e da África Sub-Saara, a OMS desenvolveu um instrumento de avaliação da qualidade de vida direcionado a esse público. O instrumento foi concebido baseado na premissa de que um trabalho multidisciplinar, envolvendo centros situados em vários países, possibilitaria uma maior difusão do produto desenvolvido. ${ }^{3}$

O WHOQOL-HIV é um módulo complementar da versão completa do instrumento do WHOQOL-100, tal qual, também foi traduzido e validado para outros idiomas em diversos estudos, entre os quais estão inseridos os de Starace et al., ${ }^{4}$ Zimpel e Fleck, ${ }^{5}$ Saddki et al., ${ }^{6}$ Canavarro et al., ${ }^{7}$ Mweemba et al. ${ }^{8}$ e Hsiung et al. ${ }^{9}$

Ainda que se trate de instrumentos de significativa difusão, dúvidas referentes ao cálculo e análise dos resultados dos instrumentos WHOQOL constituem uma limitação para a sua utilização. Nesse contexto, o objetivo do presente estudo é clarificar o mecanismo préestabelecido pelo Grupo WHOQOL-HIV para o cálculo dos escores dos instrumentos WHOQOL-HIV e WHOQOL-HIV-bref, e, adicionalmente, propor uma forma alternativa para a realização de tais cálculos.

\section{O WHOQOL-100}

O desenvolvimento do WHOQOL-100 foi realizado em 15 centros simultaneamente, sediados em 14 países. Após o desenvolvimento do projeto WHOQOL, novos centros foram incorporados. De acordo o WHO Field Centre for the Study of Quality of Life, ${ }^{10}$ atualmente os instrumentos WHOQOL estão disponíveis em mais de 50 idiomas.

A metodologia de desenvolvimento do WHOQOL foi seccionada em quatro grandes etapas: clarificação do conceito de qualidade de vida, estudo piloto qualitativo, desenvolvimento de um piloto, e, por fim, uma aplicação de campo. Para os centros integrados, após a conclusão da elaboração do instrumento, foi estabelecida uma metodologia que consistia na sua tradução, preparação do teste piloto, desenvolvimento das escalas de respostas e administração do piloto. ${ }^{11}$ 
Todas as questões do WHOQOL-100 são fechadas. É utilizada uma escala de respostas do tipo Likert, composta por cinco elementos, variando entre 1 e 5. Esses extremos representam $0 \%$ e $100 \%$, respectivamente. Existem quatro tipos diferentes de escala de respostas, conforme pode ser observado no Quadro 1:

Quadro 1 - Escalas de respostas do WHOQOL-100

\begin{tabular}{cccccc}
\hline ESCALA & $\mathbf{0 \%}$ & $\mathbf{2 5 \%}$ & $\mathbf{5 0 \%}$ & $\mathbf{7 5 \%}$ & $\mathbf{1 0 0 \%}$ \\
\hline INTENSIDADE & nada & muito pouco & mais ou menos \\
muito & insatisfeito & bem satisfeito & bante & extremamente \\
& nem insatisfeito & satisfeito & muito satisfeito \\
AVALIAÇÃO & muito ruim & ruim & $\begin{array}{c}\text { nem ruim } \\
\text { nem bom }\end{array}$ & bom & muito bom \\
& muito & infeliz & nem feliz & feliz & muito feliz \\
CAPACIDADE & nadeliz & muito pouco & médio & muito & completamente \\
FREQÜÊNCIA & nunca & raramente & às vezes & repetidamente & sempre \\
\hline
\end{tabular}

Fonte: Adaptado de Grupo WHOQOL, 1998.

O WHOQOL-100 objetiva avaliar a qualidade de vida de forma global, a partir de seis grandes domínios: Físico, Psicológico, Nível de independência, Relações sociais, Ambiente e Aspectos espirituais/Religião/Crenças pessoais. Para a obtenção dos resultados das aplicações dos instrumentos WHOQOL, o Grupo WHOQOL recomenda o software Statistical Package for the Social Sciences (SPSS).

\subsection{Cálculo do escore do WHOQOL-100}

Os resultados da aplicação do WHOQOL-100 são expressos através dos escores de cada faceta e domínio. O procedimento de pontuação do WHOQOL-100 segue a seguinte lógica:

-É verificado se todas as 100 questões foram preenchidas com valores entre 1 e 5;

- Invertem-se as 18 questões cuja escala de respostas é invertida;

- Os escores das facetas são calculados a partir da média aritmética simples das questões que compõem cada faceta, seguido de uma multiplicação por quatro. Serão computadas somente as facetas que possuírem pelo menos três itens válidos; 
- São calculados os escores dos domínios através da média aritmética simples entre os escores das facetas que compõem cada domínio. Nos domínios compostos por até cinco facetas, este será calculado somente se o número de facetas não calculadas não for igual ou superior a dois. Nos domínios compostos por mais de cinco facetas, este será calculados somente se o número de facetas não calculadas não for igual ou superior a três. No caso de facetas em escala invertida (todas as questões pertencentes à faceta possuem escala de respostas invertida), realiza-se a inversão dessa faceta para o prosseguimento do cálculo;

- Os escores dos domínios e facetas são convertidos para uma escala de 0 a 100;

- Realiza-se uma contagem do total de itens respondidos por cada respondente. São computados no cálculo somente os respondentes que preencheram corretamente pelo menos 80 itens ( $80 \%$ dos itens do instrumento).

Os resultados do WHOQOL-100 são expressos em duas escalas. Uma escala variante entre 4 e 20 pontos, devido ao fato do cálculo do escore das facetas ser realizado através da multiplicação da média das questões que constituem cada faceta por quatro. Como cada domínio é calculado através da média aritmética simples das facetas que o compõem, os resultados são expressos na mesma escala das facetas. Os resultados são expressos, também, em uma escala de 0 a 100.

\subsection{Conversão da escala de respostas das questões e facetas}

A inversão das questões é utilizada com o objetivo de padronizar todas as respostas do instrumento, de forma que a resposta mais positiva seja 5. Por conseguinte, a resposta mais negativa deve ser 1 . Desta forma, todas as questões de cada faceta são convertidas para uma mesma escala, onde o aumento gradativo da resposta equivale, na mesma proporção, ao aumento no positivismo do resultado da faceta.

Nos casos em que todas as quatro questões que constituem uma faceta sejam dispostas em escala invertida, essa mesma lógica é utilizada, mas somente no cálculo do domínio. Ou seja, o resultado dessas facetas é expresso na escala original, sem inversão (quanto mais próximo de 1, mais positivo é o resultado, e quanto mais próximo de 5, mais negativo é o

Conexões: revista da Faculdade de Educação Física da UNICAMP, Campinas, v. 10, n. 1, p. 50-69, jan./abr. 2012. 
resultado). Entretanto, ao se calcular o escore dos domínios nos quais tais facetas estão inseridas, o escore dessas é invertido.

Para a inversão da escala de resposta das questões, o valor mínimo da questão de escala invertida deve ser substituído pelo valor máximo da questão de escala normal, assim como o valor máximo da questão de escala invertida deve ser substituído pelo valor mínimo da questão de escala normal. O mesmo deve ocorrer com os valores intermediários, seguindo essa mesma lógica. Assim, o único valor que não é alterado é o valor central, que tanto na escala normal, quanto na escala invertida, permanece o mesmo.

É preciso estar atento a esse fato, pois ao comparar os resultados entre as facetas, o escore de uma faceta de escala invertida não pode ser diretamente comparado ao escore de uma faceta de escala normal. Para a conversão de uma questão de escala invertida para a escala normal, deve-se subtrair a resposta da questão de seis (6) unidades. Pode se observar no Quadro 2 os valores assumidos pelas respostas de questões cuja escala é invertida:

Quadro 2 - Conversão de escala

\begin{tabular}{lccccc}
\hline \multicolumn{1}{c}{ ESCALA } & $\mathbf{0 \%}$ & $\mathbf{2 5 \%}$ & $\mathbf{5 0 \%}$ & $\mathbf{7 5 \%}$ & $\mathbf{1 0 0 \%}$ \\
\hline Normal & 1 & 2 & 3 & 4 & 5 \\
Invertida & 5 & 4 & 3 & 2 & 1 \\
\hline
\end{tabular}

Já no caso das facetas, o escore da faceta invertida deve ser subtraído de 24 unidades. Portanto, após a conversão de uma faceta negativa, os valores 4, 8, 12, 16 e 20 passam a assumir os valores $20,16,12,8$ e 4 , respectivamente.

\subsection{Questões, domínios e facetas do WHOQOL-100}

Composto por 100 questões, o WHOQOL-100 é seccionado em 24 grupos de quatro questões cada, recebendo a denominação de "facetas". Por sua vez, o conjunto de facetas constitui um "domínio". Ao contrário da composição das facetas, os seis domínios do WHOQOL-100 não são constituídos pelo mesmo número de facetas, podendo variar de um até oito facetas. 
As questões que compõem o WHOQOL-100 não estão dispostas no questionário seguindo uma sequiência lógica por domínio ou por faceta, estão agrupadas por tipo de escala de respostas. A distribuição das facetas e domínios do WHOQOL-100 está relacionada no Quadro 3:

Quadro 3 - Domínios e facetas do WHOQOL-100

\begin{tabular}{|c|c|}
\hline DOMÍNIOS & FACETAS \\
\hline \multirow{3}{*}{ Domínio I - Domínio físico } & 1. Dor e desconforto \\
\hline & 2. Energia e fadiga \\
\hline & 3. Sono e repouso \\
\hline \multirow{5}{*}{ Domínio II - Domínio psicológico } & 4. Sentimentos positivos \\
\hline & 5. Pensar, aprender, memória e concentração \\
\hline & 6. Auto-estima \\
\hline & 7. Imagem corporal e aparência \\
\hline & 8. Sentimentos negativos \\
\hline \multirow{4}{*}{ Domínio III - Nível de Independência } & 9. Mobilidade \\
\hline & 10. Atividades da vida cotidiana \\
\hline & 11. Dependência de medicação ou de tratamentos \\
\hline & 12. Capacidade de trabalho \\
\hline \multirow{3}{*}{ Domínio IV - Relações sociais } & 13. Relações pessoais \\
\hline & 14. Suporte (Apoio) social \\
\hline & 15. Atividade sexual \\
\hline \multirow{8}{*}{ Domínio V- Ambiente } & 16. Segurança física e proteção \\
\hline & 17. Ambiente no lar \\
\hline & 18. Recursos financeiros \\
\hline & 19. Cuidados de saúde e sociais: disponibilidade e qualidade \\
\hline & 20. Oportunidades de adquirir novas informações e habilidades \\
\hline & 21. Participação em, e oportunidades de recreação/lazer \\
\hline & 22. Ambiente físico: (poluição/ruído/trânsito/clima) \\
\hline & 23. Transporte \\
\hline $\begin{array}{l}\text { Domínio VI- Aspectos } \\
\text { espirituais/Religião/Crenças pessoais }\end{array}$ & 24. Espiritualidade/religião/crenças pessoais \\
\hline
\end{tabular}
Fonte: Grupo WHOQOL, 1998.

O WHOQOL-100 possui uma faceta que não está inserida em nenhum domínio, a faceta Qualidade de vida global e percepção geral da saúde. ${ }^{11}$ Esta faceta aborda uma autoavaliação da qualidade de vida, em que o respondente expressa o seu ponto de vista de satisfação com a sua vida, saúde e qualidade de vida.

\subsection{Versão abreviada do WHOQOL-100 (WHOQOL-bref)}

Com o objetivo de disponibilizar um instrumento que demande menor tempo para preenchimento e com características psicométricas satisfatórias, o Grupo WHOQOL desenvolveu a versão abreviada do WHOQOL-100, o WHOQOL-bref . ${ }^{12}$ 
O WHOQOL-bref é composto por 26 questões, sendo duas questões sobre a auto-avaliação da qualidade de vida e 24 questões representando cada uma das facetas do WHOQOL-100. Para a composição das questões do WHOQOL-bref foi selecionada a questão de cada faceta que apresentava a maior correlação com o escore médio de todas as facetas. ${ }^{13}$

Após a seleção das questões, foi realizada uma análise para verificar se estas, factualmente, representavam as facetas correspondentes. Em seis facetas, a questão selecionada foi substituída por outra questão da faceta correspondente, pois, sob o viés de experts, havia outra questão que melhor definisse essas seis facetas. ${ }^{13}$ As facetas pertencentes ao domínio Nível de Independência foram incorporadas ao domínio Físico, assim como a faceta pertencente ao domínio Aspectos espirituais/Religião/Crenças pessoais foi incorporada ao domínio Psicológico. Assim, o WHOQOL-bref é composto por quatro domínios: Físico, Psicológico, Relações Sociais e Meio-Ambiente, perfazendo configuração expressa no Quadro 4:

Quadro 4 - Domínios e facetas do WHOQOL-bref

\begin{tabular}{|c|c|}
\hline DOMÍNIOS & FACETAS \\
\hline \multirow{7}{*}{ Domínio I - Domínio físico } & 1. Dor e desconforto \\
\hline & 2. Energia e fadiga \\
\hline & 3. Sono e repouso \\
\hline & 4. Mobilidade \\
\hline & 5. Atividades da vida cotidiana \\
\hline & 6. Dependência de medicação ou de tratamentos \\
\hline & 7. Capacidade de trabalho \\
\hline \multirow{6}{*}{ Domínio II - Domínio psicológico } & 8. Sentimentos positivos \\
\hline & 9. Pensar, aprender, memória e concentração \\
\hline & 10. Auto-estima \\
\hline & 11. Imagem corporal e aparência \\
\hline & 12. Sentimentos negativos \\
\hline & 13. Espiritualidade/religião/crenças pessoais \\
\hline \multirow{3}{*}{ Domínio III - Relações sociais } & 14. Relações pessoais \\
\hline & 15. Suporte (Apoio) social \\
\hline & 16. Atividade sexual \\
\hline \multirow{8}{*}{ Domínio IV- Meio-Ambiente } & 17. Segurança física e proteção \\
\hline & 18. Ambiente no lar \\
\hline & 19. Recursos financeiros \\
\hline & 20. Cuidados de saúde e sociais: disponibilidade e qualidade \\
\hline & 21. Oportunidades de adquirir novas informações e habilidades \\
\hline & 22. Participação em, e oportunidades de recreação/lazer \\
\hline & 23. Ambiente físico: (poluição/ruído/trânsito/clima) \\
\hline & 24. Transporte \\
\hline
\end{tabular}

Fonte: The WHOQOL Group, 1998b. 
O cálculo dos escores do WHOQOL-bref segue a mesma lógica do WHOQOL-100, exceto pelo cálculo dos escores das facetas. No WHOQOL-bref cada faceta é representada por uma única questão, e, por esse motivo, os escores das facetas não são calculados. ${ }^{12}$

\section{WHOQOL-HIV}

Com o objetivo de criar um instrumento de avaliação da qualidade de vida direcionado para portadores do HIV, pesquisadores da Joint United Nations Programme on HIV/AIDS (UNAIDS) e da OMS realizaram estudos em portadores do HIV em nove diferentes países. O resultado desse estudo foi o instrumento WHOQOL-HIV, um módulo adicional específico para pessoas portadoras do HIV ou AIDS. ${ }^{10}$

O WHOQOL-HIV avalia a qualidade de vida a partir de seis domínios e 29 facetas. Os domínios e facetas são os mesmos do WHOQOL-100, com o acréscimo de cinco facetas específicas para portadores do HIV. A faceta do WHOQOL-100 que avalia a qualidade de vida do ponto de vista do avaliado, não inserida em nenhum domínio, permanece no WHOQOL-HIV. As facetas específicas para os portadores do HIV, assim como as facetas do WHOQOL-100, são compostas por quatro questões. ${ }^{14}$ As facetas adicionais do WHOQOL-HIV são:

- Sintomas de pessoas vivendo com HIV/AIDS (PVHAs): problemas físicos que PVHAs podem vir a apresentar;

- Inclusão social: aceitação do indivíduo pela sociedade em que está inserido;

- Perdão e culpa: sentimento de culpa que o indivíduo possui a respeito da sua infecção pelo HIV;

- Preocupações sobre o futuro: medo e preocupações sobre as mudanças no curso da vida do indivíduo após a infecção pelo HIV;

- Morte e morrer: preocupações com a morte, tal qual o local, o motivo e o sofrimento antes de morrer.

As facetas adicionais do WHOQOL-HIV se inserem nos domínios já existentes do WHOQOL-100, como pode ser observado no Quadro 5: 
Quadro 5 - Domínios e facetas exclusivas do WHOQOL-HIV

\begin{tabular}{ll}
\hline \multicolumn{1}{c}{ DOMÍNIOS } & \multicolumn{1}{c}{ FACETAS } \\
\hline Domínio I - Físico & 50. Sintomas de PVHAs \\
Domínio IV - Relações sociais & 51. Inclusão social \\
Domínio VI - Aspectos & 52. Perdão e culpa \\
espirituais/Religião/Crenças pessoais & 53. Preocupações sobre o futuro \\
& 54. Morte e morrer \\
\hline
\end{tabular}

Fonte: Adaptado de O'CONNELL et al., 2004.

Tendo por base a configuração supracitada, as questões que compõem as facetas adicionais do WHOQOL-HIV, com as questões invertidas grafadas em itálico, são:

\section{Quadro 6 - Questões adicionais do WHOQOL-HIV}

\begin{tabular}{|c|c|}
\hline FACETAS & QUESTÕES \\
\hline \multirow{4}{*}{ Sintomas de PVHAs } & $\begin{array}{l}\text { O quanto você fica incomodado por ter (ou ter tido) algum problema físico } \\
\text { desagradável relacionado à sua infecção por HIV? }\end{array}$ \\
\hline & Em que medida você tem medo de uma possível dor (física) no futuro? \\
\hline & $\begin{array}{l}\text { Em que medida você sente que qualquer problema físico desagradável o impede de } \\
\text { fazer coisas que são importantes para você? }\end{array}$ \\
\hline & $\begin{array}{l}\text { Em que medida você se sente incomodado pelo medo de vir a ter qualquer problema } \\
\text { físico desagradável? }\end{array}$ \\
\hline \multirow{4}{*}{ Inclusão social } & Em que medida você se sente aceito pelas pessoas que você conhece? \\
\hline & Com que freqüência você sente que é discriminado devido à sua condição de saúde? \\
\hline & Em que medida você se sente aceito pela sua comunidade? \\
\hline & $\begin{array}{l}\text { O quanto você se sente afastado, alheio, distante emocionalmente dos outros e das } \\
\text { pessoas que lhe rodeiam? }\end{array}$ \\
\hline \multirow{4}{*}{ Perdão e culpa } & O quanto você se culpa pela sua infecção pelo HIV? \\
\hline & $\begin{array}{l}\text { Você se incomoda com o fato das pessoas lhe responsabilizarem pela sua condição } \\
\text { de HIV? }\end{array}$ \\
\hline & Quão culpado você se sente por ser HIV+? \\
\hline & $\begin{array}{l}\text { Em que medida você se sente culpado quando necessita da ajuda ou do cuidado dos } \\
\text { outros? }\end{array}$ \\
\hline \multirow{3}{*}{$\begin{array}{l}\text { Preocupações sobre } \\
\text { o futuro }\end{array}$} & $\begin{array}{l}\text { Em que medida você se preocupa que a sua condição de HIV interrompa a } \\
\text { possibilidade de continuar a sua família e as futuras gerações? }\end{array}$ \\
\hline & $\begin{array}{l}\text { Em que medida você está preocupado em como as pessoas lembrarão de vocêe } \\
\text { quando morrer? }\end{array}$ \\
\hline & $\begin{array}{l}\text { Em que medida lhe incomoda sentir que seu sofrimento é determinado pelo destino? } \\
\text { O quanto você tem medo do futuro? }\end{array}$ \\
\hline \multirow{4}{*}{ Morte e morrer } & O quanto você se preocupa com a morte? \\
\hline & $\begin{array}{l}\text { Quão perturbado você está com a idéia de não poder morrer da maneira como você } \\
\text { gostaria? }\end{array}$ \\
\hline & Quão preocupado você está em relação a como e onde você morrerá? \\
\hline & Quão preocupado você está em relação à possibilidade de sofrer antes de morrer? \\
\hline
\end{tabular}

A sintaxe para o cálculo dos escores dos domínios e facetas do WHOQOL-HIV, com correção ao erro reportado por Pedroso et al., ${ }^{15}$ é a seguinte: 
Quadro 7 - Sintaxe SPSS para o cálculo dos escores do WHOQOL-HIV

\section{ETAPAS SINTAXE SPSS PARA O CÁLCULOS DOS ESCORES DO WHOQOL-HIV}

Verificar se todos os 120 itens foram preenchidos com respostas entre 1 e 5

Converter as questões invertidas
RECODE F11 F12 F13 F14 F21 F22 F23 F24 F31 F32 F33 F34 F501 F502 F503 F504 F41 F42 F43 F44 F51 F52 F53 F54 F61 F62 F63 F64 F71 F72 F73 F74 F81 F82 F83 F84 F91 F92 F93 F94 F101 F102 F103 F104 F111 F112 F113 F114 F121 F122 F123 F124 F131 F132 F133 F134 F141 F142 F143 F144 F151 F152 F153 F154 F511 F512 F513 F514 F161 F162 F163 F164 F171 F172 F173 F174 F181 F182 F183 F184 F191 F192 F193 F194 F201 F202 F203 F204 F211 F212 F213 F214 F221 F222 F223 F224 F231 F232 F233 F234 F241 F242 F243 F244 F521 F522 F523 F524 F531 F532 F533 F534 F541 F542 F543 F544 G1 G2 G3 G4 (1=1) $(2=2)(3=3)(4=4)(5=5)($ ELSE=SYSMIS).

RECODE F11 F12 F13 F14 F22 F24 F32 F34 F72 F73 F81 F82 F83 F84 F93 F94 F102 F104 F111 F112 F113 F114 F131 F154 F163 F182 F184 F222 F232 F234 F501 F502 F503 F504 F514 F512 F521 F522 F523 F524 F531 F532 F533 F534 F541 F542 F544 F543 (1=5) (2=4) $(3=3)(4=2)(5=1)(1=5)(2=4)(3=3)(4=2)(5=1)$. COMPUTE PAIN $=(\mathrm{F} 11+\mathrm{F} 12+\mathrm{F} 13+\mathrm{F} 14) / 4$. COMPUTE ENERGY $=(\mathrm{F} 21+\mathrm{F} 22+\mathrm{F} 23+\mathrm{F} 24) / 4$. COMPUTE SLEEP $=(\mathrm{F} 31+\mathrm{F} 32+\mathrm{F} 33+\mathrm{F} 34) / 4$. COMPUTE SYMPTOM=(F501+F502+F503+F504)/4. COMPUTE PFEEL $=(\mathrm{F} 41+\mathrm{F} 42+\mathrm{F} 43+\mathrm{F} 44) / 4$. COMPUTE COG $=(\mathrm{F} 51+\mathrm{F} 52+\mathrm{F} 53+\mathrm{F} 54) / 4$. COMPUTE ESTEEM= $(\mathrm{F} 61+\mathrm{F} 62+\mathrm{F} 63+\mathrm{F} 64) / 4$. COMPUTE BODY $=(\mathrm{F} 71+\mathrm{F} 72+\mathrm{F} 73+\mathrm{F} 74) / 4$. COMPUTE NFEEL $=(\mathrm{F} 81+\mathrm{F} 82+\mathrm{F} 83+\mathrm{F} 84) / 4$. COMPUTE MOBIL $=(\mathrm{F} 91+\mathrm{F} 92+\mathrm{F} 93+\mathrm{F} 94) / 4$. COMPUTE ADL $=(\mathrm{F} 101+\mathrm{F} 102+\mathrm{F} 103+\mathrm{F} 104) / 4$. COMPUTE DEPEND $=(\mathrm{F} 111+\mathrm{F} 112+\mathrm{F} 113+\mathrm{F} 114) / 4$. COMPUTE WORK= $(\mathrm{F} 121+\mathrm{F} 122+\mathrm{F} 123+\mathrm{F} 124) / 4$. COMPUTE RELATIO $=(\mathrm{F} 131+\mathrm{F} 132+\mathrm{F} 133+\mathrm{F} 134) / 4$. COMPUTE SUPPORT $=(\mathrm{F} 141+\mathrm{F} 142+\mathrm{F} 143+\mathrm{F} 144) / 4$. COMPUTE SEX=(F151+F152+F153+F154)/4. COMPUTE INCLUSI=(F511+F512+F513+F514)/4. COMPUTE SAFE $=(\mathrm{F} 161+\mathrm{F} 162+\mathrm{F} 163+\mathrm{F} 164) / 4$. COMPUTE HOME $=(\mathrm{F} 171+\mathrm{F} 172+\mathrm{F} 173+\mathrm{F} 174) / 4$. COMPUTE FINANCE $=(\mathrm{F} 181+\mathrm{F} 182+\mathrm{F} 183+\mathrm{F} 184) / 4$. COMPUTE CARE $=(\mathrm{F} 191+\mathrm{F} 192+\mathrm{F} 193+\mathrm{F} 194) / 4$. COMPUTE INFO=(F201+F202+F203+F204)/4. COMPUTE LEISURE $=(\mathrm{F} 211+\mathrm{F} 212+\mathrm{F} 213+\mathrm{F} 214) / 4$. COMPUTE ENVIRO=(F221+F222+F223+F224)/4. COMPUTE TRANS $=(\mathrm{F} 231+\mathrm{F} 232+\mathrm{F} 233+\mathrm{F} 234) / 4$. COMPUTE SRPB $=(\mathrm{F} 241+\mathrm{F} 242+\mathrm{F} 243+\mathrm{F} 244) / 4$. COMPUTE FORGIVE $=(\mathrm{F} 521+\mathrm{F} 522+\mathrm{F} 523+\mathrm{F} 524) / 4$. COMPUTE FUTURE=(F531+F532+F533+F534)/4. COMPUTE DEATH=(F541+F542+F543+F544)/4. COMPUTE GENERAL $=(\mathrm{G} 1+\mathrm{G} 2+\mathrm{G} 3+\mathrm{G} 4) / 4$. COMPUTE DOMAIN1 $=($ PAIN+ENERGY+SLEEP+SYMPTOM $) / 4 * 4$. COMPUTE DOMAIN2 $=(\mathrm{PFEEL}+\mathrm{COG}+\mathrm{ESTEEM}+\mathrm{BODY}+\mathrm{NFEEL}) / 5 * 4$. COMPUTE DOMAIN3=(MOBIL+ADL+DEPEND+WORK $) / 4 * 4$. COMPUTE DOMAIN4=(RELATIO+SUPPORT+SEX+INCLUSI $) / 4 * 4$. COMPUTE DOMAIN5=(SAFE+HOME+FINANCE+CARE+INFO+LEISURE+ENVIRO+TRANS $) / 8 * 4$. COMPUTE DOMAIN6=(FORGIVE+FUTURE+DEATH+SRPB $) / 4 * 4$.

Fonte: Adaptado de The WHOQOL-HIV Group, 2002. 
O cálculo dos resultados do WHOQOL-HIV é similar ao do WHOQOL-100. No entanto, alguns critérios utilizados no WHOQOL-100 não foram herdados pelo WHOQOL-HIV. Os resultados do WHOQOL-HIV são calculados a partir da seguinte lógica:

- É verificado se todas as 120 questões foram preenchidas com valores entre 1 e 5;

- Invertem-se todas as questões cuja escala de respostas é invertida. Em se tratando de facetas em escala invertida, todas as questões pertencentes a estas facetas são invertidas individualmente;

- Os escores das facetas são calculados a partir da soma das quatro questões pertencentes a cada faceta, seguido de uma divisão por quatro. Estes são expressos em uma escala variante entre 1 e 5;

- Os escores dos domínios são calculados através da soma dos escores das "n" facetas que compõem cada domínio, dividido pelo número de facetas do domínio. O resultado é multiplicado por quatro, sendo estes expressos em uma escada de 4 a 20.

Ao contrário do que ocorre no WHOQOL-100, o escore dos domínios e facetas representam a média destes, unicamente, quando todos os itens pertencentes a estes são corretamente pontuados. O escore das facetas é calculado desde que estas possuam pelo menos uma questão respondida, enquanto o escore dos domínios é calculado desde que estes possuam minimamente uma faceta que tenha sido pontuada. Estes não são convertidos para uma escala de 0 a 100. O critério de exclusão dos indivíduos que responderam incorretamente ou deixaram de responder mais do que $20 \%$ do total de questões do instrumento não se faz presente na sintaxe do WHOQOL-HIV.

\section{WHOQOL-HIV-bref}

Sob a mesma justificativa da elaboração do WHOQOL-bref, o Grupo WHOQOL desenvolveu uma versão abreviada do WHOQOL-HIV. O WHOQOL-HIV-bref é baseado no WHOQOL-bref, da forma que cada faceta é representada por uma única questão.

As 26 questões do WHOQOL-bref se repetem no WHOQOL-HIV-bref, sendo estas acrescidas de cinco questões que representam as facetas adicionais do WHOQOL-HIV. ${ }^{16}$ Ao contrário do que ocorre no WHOQOL-bref, as facetas pertencentes aos domínios nível

Conexões: revista da Faculdade de Educação Física da UNICAMP, Campinas, v. 10, n. 1, p. 50-69, jan./abr. 2012. 
de independência e Aspectos espirituais/Religião/Crenças pessoais não são incorporadas aos domínios Físico e Psicológico, assumindo, assim, a mesma configuração dos domínios do WHOQOL-HIV, apresentando-se sob a seguinte configuração:

\section{Quadro 8 - Questões do WHOQOL-HIV-bref}

\section{DOMÍNIOS}

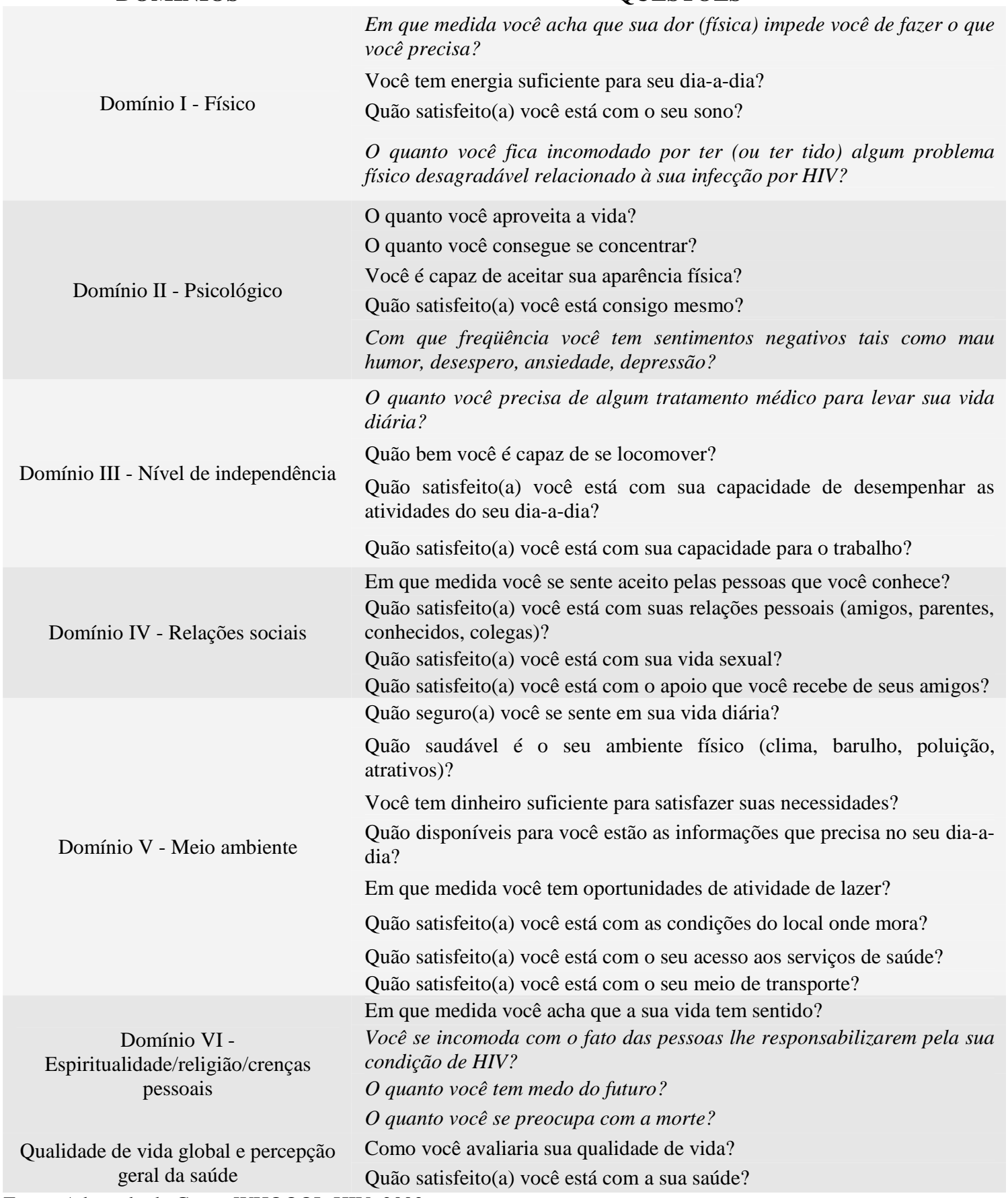

Fonte: Adaptado de Grupo WHOQOL-HIV, 2002. 
O cálculo dos escores do WHOQOL-HIV-bref, por sua vez, segue uma lógica diferenciada da presente no WHOQOL-bref, consistindo dos seguintes comandos:

Quadro 9 - Sintaxe SPSS para cálculo dos escores do WHOQOL-HIV-bref

\begin{tabular}{|c|c|}
\hline ETAPAS & SINTAXE SPSS PARA O CÁLCULOS DOS ESCORES DO WHOQOL-HIV-BREF \\
\hline $\begin{array}{l}\text { Verificar se } \\
\text { todos os } 31 \\
\text { itens foram } \\
\text { preenchidos } \\
\text { com } \\
\text { respostas } \\
\text { entre } 1 \text { e } 5\end{array}$ & $\begin{array}{l}\text { RECODE Q1 Q2 Q3 Q4 Q5 Q6 Q7 Q8 Q9 Q10 Q11 Q12 Q13 Q14 Q15 Q16 Q17 Q18 Q19 } \\
\text { Q20 Q21 Q22 Q23 Q24 Q25 Q26 Q27 Q28 Q29 Q30 Q31 }(1=1)(2=2)(3=3)(4=4)(5=5) \\
\text { (ELSE=SYSMIS). }\end{array}$ \\
\hline $\begin{array}{l}\text { Converter as } \\
\text { questões } \\
\text { invertidas }\end{array}$ & RECODE Q3 Q4 Q5 Q8 Q9 Q10 Q31 (1=5) (2=4) (3=3) (4=2) (5=1). \\
\hline $\begin{array}{l}\text { Calcular os } \\
\text { escores dos } \\
\text { domínios }\end{array}$ & $\begin{array}{l}\text { COMPUTE Domain } 1=(\mathrm{Q} 3+\mathrm{Q} 4+\mathrm{Q} 14+\mathrm{Q} 21) / 4 * 4 \\
\text { COMPUTE Domain } 2=(\mathrm{Q} 6+\mathrm{Q} 11+\mathrm{Q} 15+\mathrm{Q} 24+\mathrm{Q} 31) / 5 * 4 \\
\text { COMPUTE Domain } 3=(\mathrm{Q} 5+\mathrm{Q} 22+\mathrm{Q} 23+\mathrm{Q} 20) / 4 * 4 \\
\text { COMPUTE Domain } 4=(\mathrm{Q} 27+\mathrm{Q} 26+\mathrm{Q} 25+\mathrm{Q} 17) / 4 * 4 \\
\text { COMPUTE Domain } 5=(\mathrm{Q} 12+\mathrm{Q} 13+\mathrm{Q} 16+\mathrm{Q} 18+\mathrm{Q} 19+\mathrm{Q} 28+\mathrm{Q} 29+\mathrm{Q} 30) / 8 * 4 \\
\text { COMPUTE Domain6 }=(\mathrm{Q} 7+\mathrm{Q} 8+\mathrm{Q} 9+\mathrm{Q} 10) / 4 * 4\end{array}$ \\
\hline
\end{tabular}

Fonte: The WHOQOL-HIV Group, 2002.

A transcrição textual da sintaxe do WHOQOL-HIV-bref se apresenta da seguinte forma:

-É verificado se todas as 31 questões foram preenchidas com valores entre 1 e 5;

- Invertem-se todas as questões cuja escala de respostas é invertida;

- Os escores dos domínios são calculados através da soma dos escores das "n" questões que compõem cada domínio, dividido pelo número de questões do domínio. O resultado é multiplicado por quatro, sendo representado em uma escala de 4 a 20.

Como pode ser percebido, tal qual o WHOQOL-HIV, a sintaxe do WHOQOL-HIV-bref apresenta a mesma fragilidade presente no WHOQOL-HIV com relação ao cálculo dos escores dos domínios e facetas, pelo fato de não se realizar a média aritmética dos componentes do domínio. Não há a conversão dos escores dos domínios e facetas para uma escala de 0 a 100, e, também, não é previsto o critério de eliminação dos respondentes que deixaram de responder ou responderam incorretamente um número de questões superior a $20 \%$ do total de questões do instrumento. 


\section{FERRAMENTAS PARA O CÁlCULO DOS ESCORES E ESTATÍSTICA DESCRITIVA DOS INSTRUMENTOS WHOQOL-HIV E WHOQOL-HIV-BREF}

Para a obtenção dos resultados das aplicações dos instrumentos WHOQOL, o Grupo WHOQOL recomenda a utilização do software SPSS, um software estatístico que exige conhecimentos específicos para a sua utilização e não é de distribuição gratuita.

Almejando a supressão dessas limitações, foram construídas ferramentas, a partir do software Microsoft Excel - um software de ampla acessibilidade -, para a realização do cálculo dos escores e estatística descritiva dos instrumentos WHOQOL-HIV e WHOQOLHIV-bref. Tais ferramentas foram confeccionadas nos mesmos moldes das ferramentas desenvolvidas por Pedroso, Pilatti e Reis, ${ }^{17}$ Pedroso et al., ${ }^{18}$ Pedroso, Pilatti e Gutierrez, ${ }^{19}$ e Pedroso et al., ${ }^{20}$ para o cálculo dos escores e estatística descritiva dos instrumento WHOQOL-100, WHOQOL-bref, WHOQOL-OLD e WHOQOL-SRPB, respectivamente.

As ferramentas propostas realizam automaticamente todos os cálculos incipientes nas sintaxes disponibilizadas pelo Grupo WHOQOL-HIV, sendo que o pesquisador que utilizá-las precisa apenas preencher as respostas concedidas pelos respondentes nas células especificadas.

Após a inserção dos dados, para a utilização dos resultados de sua pesquisa, o pesquisador poderá copiar os escores individuais de cada respondente, os resultados da estatística descritiva e os gráficos, entretanto, não pode modificar tais resultados. A única área que lhe é permitida a inserção e edição de valores é a área de tabulação das respostas dos respondentes.

Para a validação da ferramenta, foram realizadas simulações com dados reais de aplicações de cada um dos instrumentos WHOQOL-HIV e WHOQOL-HIV-bref, comparando os resultados obtidos através das ferramentas propostas com os obtidos no SPSS. Os resultados retornados por ambos os softwares foram exatamente os mesmos, garantindo assim a fidedignidade das ferramentas objeto de estudo deste trabalho. 
As ferramentas foram testadas em diferentes versões desse software - 2000, XP, 2003 e 2007. Constatou-se a sua compatibilidade com todas as versões testadas, sem divergências nos resultados. O download das ferramentas pode ser realizado através do sítio: http://www.brunopedroso.com.br/whoqol-hiv.html.

\section{CONSIDERAÇÕES FINAIS}

Ainda que os instrumentos WHOQOL-HIV e WHOQOL-HIV-bref sejam, respectivamente, módulos complementares dos instrumentos WHOQOL-100 e WHOQOLbref, a sintaxe destes instrumentos não deriva por completo das sintaxes dos seus precursores. Não obstante à ampla difusão e utilização dos instrumentos WHOQOL-HIV, a dificuldade na interpretação das sintaxes dos instrumentos constitui um fator limitante na opção por utilização de tais instrumentos.

De forma adicional, a interposição do Grupo WHOQOL em confeccionar as sintaxes para os cálculos dos escores dos instrumentos WHOQOL através do SPSS, um software de custo relativamente elevado e que exige conhecimentos específicos para a sua utilização, fomenta outro imbróglio que limita a utilização de tais instrumentos.

Fronteado a esse embate, a presente investigação realiza uma abordagem acerca dos instrumentos em exame, no intuito de facilitar a sua interpretação e utilização. Almejando a supressão das limitações supracitadas, as sintaxes são transcritas textualmente, detalhando-se todos os passos utilizados para a obtenção dos resultados dos instrumentos WHOQOL-HIV e WHOQOL-HIV-bref. Foram construídas, também, ferramentas a partir do software Microsoft Excel 2003 para a realização do cálculo dos escores e estatística descritiva dos referidos instrumentos, nas quais o pesquisador encarrega-se somente de tabular os dados, sendo que o restante dos cálculos é realizado de forma automatizada.

As ferramentas foram testadas e se demonstraram compatíveis nas versões 2000, XP e 2007 do Microsoft Excel. Os resultados retornados pelas ferramentas foram comparados, através de dados reais de aplicação dos instrumentos WHOQOL-HIV e WHOQOL-HIVbref, com os resultados retornados pelo SPSS, seguindo os parâmetros estabelecidos pelo Grupo WHOQOL-HIV. Os resultados obtidos foram idênticos em ambos os instrumentos. 
Conclui-se que, mesmo se tratando de instrumentos amplamente difundidos e desenvolvidos sob uma criteriosa metodologia, os instrumentos produzidos pelo Grupo WHOQOL-HIV apresentam limitações. Na expectativa de facilitar a sua utilização, foi realizada uma abordagem clarificadora com enfoque em tais instrumentos. Nessa perspectiva, objetivou-se possibilitar uma maior acessibilidade aos resultados fomentados pelos instrumentos objeto de estudo deste trabalho, perfazendo com que a investigação no entorno da realidade empírica da qualidade de vida dos portadores de HIV/AIDS seja ampliada.

\section{REFERÊNCIAS}

${ }^{1}$ SKEVINGTON, S. M.; O'CONNELL, K. A. Measuring quality of life in HIV and AIDS: a review of the recent literature. AIDS Care, Abingdon, v. 18, n. 3, p. 331-350, may/june 2003.

${ }^{2}$ FLECK, M. P. A. Problemas conceituais em qualidade de vida. In: FLECK, M. P. A. et al. (Org.). A avaliação de qualidade de vida: guia para profissionais da saúde. Porto Alegre: Artmed, 2008. p. 19-28.

${ }^{3}$ O'CONNELL, K. et al. Preliminary development of the World Health Organization's Quality of Life HIV instrument (WHOQOL-HIV): analysis of the pilot version. Social \& Science Medicine, Leicester, v. 57, n. 7, p. 1259-1275, oct. 2003.

${ }^{4}$ STARACE, F. et al. Quality of life assessment in HIV-positive persons: application and validation of the WHOQOL-HIV, Italian version. AIDS Care, Abindgon, v. 14, n. 3, p. 405-415, may/jun. 2002.

${ }^{5}$ ZIMPEL, R.; FLECK, M. P. A. Quality of life in HIV-positive Brazilians: application and validation of the WHOQOL-HIV, Brazilian version. AIDS Care, Abingdon, v. 19, n. 7, p. 923-930, aug. 2007. 
${ }^{6}$ SADDKI, N. et al. Validity and reliability of the Malay version of WHOQOL-HIV BREF in patients with HIV infection. AIDS Care, Abingdon, v. 21, n. 10, p. 1271-1278, oct. 2009.

${ }^{7}$ CANAVARRO, M. C. et al. Quality of life assessment in HIV-infection: validation of the European Portuguese version of WHOQOL-HIV. AIDS Care, Abingdon, v. 23, n. 2, p. 187-194, feb. 2011.

${ }^{8}$ MWEEMBA, P. et al. Validation of the World Health Organization Quality of Life HIV instrument in a Zambian sample. Journal of the Association of Nurses in AIDS Care, Philadelphia, v. 22, n. 1, p. 53-66, fev. 2011.

${ }^{9}$ HSIUNG, P. et al. Validation of the WHOQOL-HIV BREF among HIV-infected patients in Taiwan. AIDS Care, Abingdon, v. 23, n. 8, p. 1035-1042, ago. 2011.

${ }^{10}$ WHO FIELD CENTRE FOR THE STUDY OF QUALITY OF LIFE OF BATH. About the WHO Field Centre for the Study of Quality of Life. University of Bath, 2008. WHO Field Centre for the Study of Quality of Life. Disponível em: <http://www.bath.ac.uk/whoqol/about.cfm>. Acesso em: 13 abr. 2008.

${ }^{11}$ THE WHOQOL GROUP. WHOQOL user manual. Geneva: World Health Organization, 1998a.

${ }^{12}$ THE WHOQOL GROUP. WHOQOL-bref: introduction, administration, scoring and generic version of assessment. Geneva: World Health Organization, 1996.

${ }^{13}$ THE WHOQOL GROUP. Development of the World Health Organization WHOQOLBREF Quality of Life Assessment. Psychological Medicine, Cambridge, UK, v. 28, n. 3, p. 551-558, may 1998 b. 
${ }^{14} \mathrm{O}$ 'CONNELL, K. et al. WHOQOL-HIV for quality of life assessment among people living with HIV and AIDS: results from a field test. AIDS Care, Abingdon, v. 16, n. 7, p. 882889 , oct. 2004.

${ }^{15}$ PEDROSO, B. et al. Quality of life assessment in people with HIV: analysis of the WHOQOL-HIV syntax. AIDS Care, Abingdon, v. 22, n. 3, p. 361-372, mar. 2010a.

${ }^{16}$ THE WHOQOL-HIV GROUP. WHOQOL-HIV instrument users manual. Geneva: World Health Organization, 2002.

${ }^{17}$ PEDROSO, B.; PILATTI, L. A.; REIS, D. R. Cálculo dos escores e estatística descritiva do WHOQOL-100 utilizando o Microsoft Excel. Revista Brasileira de Qualidade de Vida, Ponta Grossa, v. 1, n. 1, p. 23-32, jan./jul. 2009.

${ }^{18}$ PEDROSO, B. et al. Cálculo dos escores e estatística descritiva do WHOQOL-bref através do Microsoft Excel. Revista Brasileira de Qualidade de Vida, Ponta Grossa, v. 2, n. 1, p. 31-36, jan./jun. 2010b.

${ }^{19}$ PEDroso, B.; PILATTI, L. A.; GUTIERREZ, G. L. Cálculo dos escores e estatística descritiva do WHOQOL-OLD através do Microsoft Excel. Geriatria \& Gerontologia, Rio de Janeiro, v. 4, n. 4, p. 214-219, out./dez. 2010.

${ }^{20}$ PEDROSO, B. et al. Qualidade de vida, espiritualidade, religião e crenças pessoais: análise do instrumento WHOQOL-SRPB. Revista Eletrônica Fafit/Facic, Itararé, v. 3, n. 1, p. 01-12, jan./jun. 2012. 